\title{
Immunotherapy for Hymenoptera venom allergy compared with real-life stings: are we doing our best?
}

Luca Pecoraro $^{1}$, Mattia Giovannini ${ }^{2}$, Francesca Mori ${ }^{2}$, Francesca Saretta ${ }^{3}$, Simona Barni ${ }^{4}$, Riccardo Castagnoli ${ }^{5}$, Stefania Arasi ${ }^{6}$, Carla Mastrorilli ${ }^{7}$, Lucia Liotti ${ }^{8}$, Lucia Caminiti ${ }^{9}$, and Elio Novembre ${ }^{2}$

${ }^{1}$ University of Verona

${ }^{2}$ Meyer Children's University Hospital

${ }^{3}$ Hospital of Palmanova, A.S.S.5 "Bassa Friulana"

${ }^{4}$ A. Meyer Children's University Hospital

${ }^{5}$ University of Pavia

${ }^{6}$ Bambino Gesù Children's Hospital IRCCS, Rome

${ }^{7}$ Paediatric Hospital Giovanni XXIII

${ }^{8}$ Senigallia Hospital

${ }^{9}$ AOU Policlinico Gaetano Martino

September 16, 2020

\section{Hosted file}

VIT_vs_real-life_stings_final - proofread.pdf available at https://authorea.com/users/359567/ articles/481461-immunotherapy-for-hymenoptera-venom-allergy-compared-with-real-lifestings-are-we-doing-our-best 